\title{
Prioritize systemic approaches for young people's mental health
}

\author{
The COVID-19 pandemic presents opportunities for transformative actions towards improving \\ children's and young people's mental health, argues Archana Basu.
}

W hen the COVID-19 pandemic was declared in March 2020, families all over the world began to establish new routines for remote schooling and family life, as many parents juggled employment transitions. We sought to maintain connection and community to cope with isolation and fear, and developed rituals for our collective and individual grief. Consistent with our experiences as caregivers and educators, and extant developmental and traumatic stress science, our efforts were geared towards re-establishing a semblance of safety and predictability. These factors are important for coping with stressors, and, when combined with emotionally attuned caregiving, can be powerful buffers for children and youth, rather than leaving them feeling overwhelmed and vulnerable to mental distress.

As expected, the impact of the pandemic intersected with existing socio-political contexts, exacerbating long-standing structural inequities and bringing renewed attention to health inequity within the United States and around the world. The implications for children coming of age when predictability and trust in public systems feel elusive will likely be complex. Even before the pandemic, the loss of trust in institutions fuelled by partisanship and misinformation campaigns, the existential threat posed by the climate crisis, and threats to a sense of identity and authenticity from daily experiences such as reality-distorting social media face filters were all problematic issues. But increasing democratization of information through online platforms and global movements - many of which are led by young people - raises questions about our societal habit patterns and prompts a re-evaluation of our approaches. Towards this end, I highlight two key points for supporting children's future mental health and well-being.

First, the pandemic presents an opportunity for transformative actions towards implementing life course approaches that recognize that there is no health without mental health. At least half of lifetime mental disorders begin in childhood, and approximately $75 \%$ are diagnosed before age 24 (https://www. nami.org/mhstats). This underscores the importance of addressing children's and young people's mental health concerns, which have increased significantly during the pandemic. However, in the United States for instance, even prior to the pandemic there was a shortage of paediatric mental health services. This is especially true for children aged 6 and under, despite a well-established evidence base showing that early life experiences, beginning prenatally, influence the development of brain architecture that provides the basis for future behavioural and emotional health, learning, and even adult productivity. Integrating mental health care as part of routine care visits in perinatal, paediatric, family medicine or internal medicine care clinics is one key approach to supporting children and their parents or other adult caregivers. Beyond healthcare settings, tiered mental health approaches including universal social-emotional curriculums for students and school staff, and increased investments in school-based mental health professionals and paraprofessionals, also need to be considered. Such approaches, along with the use of innovative digital tools and telehealth, as appropriate, can improve access and availability of children's mental health services, and help to build a culture that normalizes mental health concerns and prioritizes emotional well-being.

Second, healthcare alone cannot improve mental health without addressing social determinants of health, such as housing instability or food insecurity. For example, different zip codes and neighbourhoods in the United States are associated with profound differences in health trajectories including mental health, educational experiences, access to blue and green spaces, and recreational opportunities. Structural inequities - policies and practices that create and perpetuate disparities in wealth, education, healthcare and other opportunities - have been exacerbated in the pandemic. In the United States, Black, Latino or Hispanic, and Indigenous communities have borne the brunt of the viral disease and economic impact. However, mental-health-related discussions have often focused on individual skill-building approaches. While children and adults alike benefit from practising these techniques, mental health interventions need to be considered in the unique social, economic and cultural context for families. Thus, policies geared towards poverty alleviation, housing stability, school and digital infrastructure, or improved healthcare access are also essential to mental health concerns. Accordingly, cross-sector collaborations that address socioeconomic conditions and mental health interventions need to be planned in concert with each other. Guided by an understanding of communities' specific experiences, mental health approaches may range from a spectrum of trauma-informed approaches to daily practices to promote coping and well-being, and build on community- and family-specific strengths.

In the field of childhood development, the concept of 'felt security' is broadly described as a psychological resource that develops through consistent experiences of attuned responsiveness from parents or caregivers. Over time, these experiences are internalized to form a psychological blueprint of adaptive and positive expectations, such as the idea that stressors are not overwhelming but manageable, and of having a sense of efficacy in organizing support for oneself to cope with stressors. There are parallels to this in our experiences within society more broadly. For instance, economic security provides an assurance that stressors such as illness or unemployment can be tolerable and not derailing for a family. Similarly, during this pandemic, timely investments and policies responsive to the unique needs of 
communities can support and empower parents and communities - the bedrocks for our children - in protecting and promoting the mental health and well-being of future generations.
Archana Basu1,2四

${ }^{1}$ Harvard T.H. Chan School of Public Health, Boston, MA, USA. ${ }^{2}$ Massachusetts General Hospital, Harvard Medical School, Boston, MA, USA.

$\square \varpi_{e-m a i l: a r b a s u @ h s p h . h a r v a r d . e d u}$
Published online: 20 September 2021

https://doi.org/10.1038/s41562-021-01185-7

Competing interests

The author declares no competing interests. 